\title{
Opioid-induced or pain relief-reduced symptoms in advanced cancer patients?
}

\author{
Sebastiano Mercadante ${ }^{\mathrm{a}, \mathrm{b}, *}$, Patrizia Villari ${ }^{\mathrm{a}}$, Patrizia Ferrera ${ }^{\mathrm{a}}$, Alessandra Casuccio ${ }^{\mathrm{c}}$ \\ a Anesthesia \& Intensive Care Unit and Pain Relief \& Palliative Care Unit La Maddalena Cancer Center, \\ Via San Lorenzo Colli 312, 90146 Palermo, Italy \\ ${ }^{\mathrm{b}}$ Intensive Care and Emergency, Università di Palermo, Italy \\ ${ }^{\mathrm{c}}$ Università di Palermo, Italy
}

Received 30 June 2004; accepted 17 February 2005

Available online 28 March 2005

\begin{abstract}
Background: While opioids in increasing doses may produce adverse effects, the same adverse effects may be associated with poor pain control. Moreover, in the clinical setting symptomatic treatment and illness may balance the outcome of opioid titration. Some adverse effects may tend to disappear continuing the treatment in a long-term period.

Aims: The aim of this study was to monitor the effects of a rapid opioid titration combined with symptomatic treatment in patients with poor relief and to monitor these changes in the following period of 20 days.

Methods: A consecutive sample of 35 patients admitted to an acute Pain Relief and Palliative Care Unit were titrated with opioids, according to a department policy, allowing administration of parenteral opioids to assist opioid titration with oral or transdermal opioids.

Results: Thirty-three patients were followed up for the period of the study. Pain was adequately controlled and doses were opioid doses were stable after a mean of $40 \mathrm{~h}$. Opioid escalation index (OEI) was extremely high initially, and then progressively declined at the following study intervals. Weakness and nausea and vomiting did not change, as well as confusion and appetite. Drowsiness, constipation and dry mouth significantly increased and then did not change, although a significant decrease in drowsiness was subsequently observed. Well-being improved some weeks after opioid stabilization. In multivariate analysis, drowsiness and dry mouth were correlated to opioid doses.

Conclusion: The effects reported were often due to multiple causes. A rapid decrease in pain intensity induced by rapid opioid titration does not produce changes in weakness, nausea and vomiting, appetite. While constipation appears the most relevant problem, resistant to common symptomatic treatment, drowsiness initially produced by acute opioid dose increase and the achievement of pain relief, tends to spontaneously decrease, probably as the result of late tolerance. Improved well-being may be the late positive effect of pain relief, also influenced by the setting of home care.

(C) 2005 European Federation of Chapters of the International Association for the Study of Pain. Published by Elsevier Ltd. All rights reserved.
\end{abstract}

Keywords: Opioids; Cancer pain; Adverse effects; Prospective longitudinal study; Advanced cancer patients

\footnotetext{
${ }^{*}$ Corresponding author. Present address: Anesthesia \& Intensive Care Unit and Pain Relief \& Palliative Care Unit La Maddalena Cancer Center, Via San Lorenzo Colli 312, 90146 Palermo, Italy. Tel.: +39091 6806521/6806111; fax: +390916806906.

E-mail address: terapiadeldolore@la-maddalena.it (S. Mercadante).
}

\section{Introduction}

Pain is one of the most feared consequences of cancer. Opioids are the mainstay of cancer pain management, but successful pain management with opioids 
requires that adequate analgesia be achieved without excessive adverse effects. Controversies exist about the global effect of opioid therapy. Patients on opioids may have more symptoms than patients not taking opioids (Grond et al., 1994), most patients with severe cancer pain may develop other associated symptoms, which are commonly induced by opioids per se, and intensity of pain has been found to be associated with a low level of function and higher intensity of symptoms (Grond et al., 1994; Klepstad et al., 2000a). Pain control may indirectly improve some symptoms and other measures, such as nausea and vomiting, well-being, mood, and appetite. Fatigue and loss of appetite were correlated with pain intensity (Klepstad et al., 2000a). While opioid titration may induce nausea and vomiting, visceral pain can be associated with vegetative responses, resulting in vomiting. An improved pain control may blunt these responses and as a consequence, reduce the frequency and intensity of gastrointestinal symptoms or improve appetite. Moreover, pain control may improve mood and well-being, whereas increasing dose of opioids may reduce the functional activities, and, as a consequence mood and well-being sensation. The global picture is also inferred by the progression of disease, which may induce some symptoms or influence some parameters in a long-term period.

Several studies report that opioid treatment is complicated by adverse effects. However, these studies have evaluated cancer patients receiving long-term treatment and were not designed in order to distinguish between strictly opioid-induced symptoms and symptoms related to other causes, or to provide possible benefits of pain relief by opioids on symptom control on a short-term basis (Cherny et al., 2001; Grond et al., 1996; Mercadante, 1999). Attempts have been recently done in patients titrated with oral morphine after they had received weak opioids. However, patients were switched with a relatively low level of pain intensity; therefore it was not possible to detect the possible effects of a dramatic change in pain intensity (Klepstad et al., 2000b). The aim of this study was to evaluate the clinical changes produced by the achievement of pain control in advanced cancer patients, regardless of the opioid dose required, in the acute setting of opioid titration. The secondary outcome was to monitor these changes chronically, to establish the long-term benefits of this condition after stabilization and the influence of other factors, such as the development of tolerance to adverse effects.

\section{Patients and methods}

Thirty-five consecutive advanced cancer patients with uncontrolled pain requiring strong opioids were scheduled for a prospective longitudinal study. Patients with an expected survival less than one month and a Karnofsky status less than 50 were excluded. All patients were admitted to a Pain Relief and Palliative Care Unit and were subsequently followed at the outpatient pain clinic or at home by frequent phone contacts. Informed consent was obtained, as well as Institutional approval for the treatment, which is a standard practice in this Unit.

The type of opioids and the route of administration were continued, if considered indicated and feasible, or changed according to the clinical needs, and doses were titrated to adequate pain relief or dose-limiting toxicity. Opioids were also given by intravenous route for treating breakthrough pain episodes and to assist and facilitate the dose finding in patients receiving slow release morphine and transdermal fentanyl. Doses were changed on daily basis or more frequently, when required. For transdermal fentanyl doses could be changed on a daily basis (Korte et al., 1996). This approach was allowed by continuous and strict nurse surveillance, and by providing frequent medical rounds per day. The use of other drugs, such as adjuvants, was considered, as well as all the general treatment commonly used in palliative care (laxatives, etc.) for treating symptoms eventually occurring.

Pain intensity and well being sensation were monitored using a numerical scale from 0 to 10 . Symptoms associated to opioid therapy or commonly present in advanced cancer patients, such as nausea and vomiting, drowsiness, confusion, xerostomy and so on, were rated using a scale from 0 to 3 (not at all, slight, a lot, awful). Constipation was evaluated as follows: $0=$ stool in the previous $24 \mathrm{~h} ; 1=2$ days before; $2=3$ days before; $3=$ four or more days before, or need for clyster. These data were collected as part of clinical management on a standard sheet routinely used. For mood it was used a simple scale: $0=$ normal, $1=$ some depressed, $2=$ moderately depressed, $3=$ severely depressed; and for appetite: $0=$ normal, $1=$ lightly decreased, $2=$ moderately decreased, $3=$ absent. Symptoms were assessed by the patients, assisted by nurses. Data were recorded at admission (T0), at the end of dose titration, when pain intensity was considered acceptable by patients (less than 4/10) and opioid doses were unchanged for 24-48 h (Tx), and 10 days after (T10), and 20 days after (T20), when all patients were at home.

The pain syndromes were considered on the basis of clinical history, anatomical site of primary tumor and distant metastases, physical examination, investigations such as CT-scan, MNR and so on, when necessary. Opioid escalation index per cent $(\mathrm{OEI} \%)$ was calculated at fixed in advance intervals. This score expresses the mean increase of opioid dosage per cent from opioid starting dose (OSD), according the following formula: $[(\mathrm{OMD}-\mathrm{OSD}) / \mathrm{OSD}] /$ days $\times 100$, where $\mathrm{OMD}$ is the maximal dose of opioid. Opioid escalation index in $\mathrm{mg}$ 
(OEI $\mathrm{mg}$ ) was calculated as the mean increase of opioid dosage in $\mathrm{mg}$ using the following formula: (OMD - OSD)/days. The meaning of this score, improved by this formula, has been discussed in previous studies (Mercadante et al., 1997).

To facilitate the calculations, opioid doses were expressed as oral morphine equivalents, by using ratios commonly employed in the Unit: morphine $60 \mathrm{mg}=$ transdermal fentanyl $0.6 \mathrm{mg}=$ methadone $12 \mathrm{mg}$.

Given the open design of the study, an independent observer was asked to establish from available clinical data, if symptoms were induced by opioid escalation or there were other possible causes.

\subsection{Statistical analysis}

Frequency analysis was performed with chi-square test. The paired Wilcoxon signed-rank test and paired samples Student's $t$-test were used to compare symptom intensity and opioid dosage, respectively, at the different intervals. The one-way analysis of variance (ANOVA) and Mann-Whitney $U$ statistic test were used to evaluate difference in age groups and gender. The multivariate analysis was performed by MANOVA test. All $p$-values were two-sided and $p$-values less than 0.05 were considered statistically significant.

\section{Results}

The mean age of patients surveyed was 58.9 years (CI 95\% 54-63). Fifteen patients were women. Thirty-three patients completed the study. Two patients died before completing the study (one before T10, and the other one before T20). Death was unexpected in relation to the previous visit-phone contact or Karnofsky status. Pain mechanisms were somatic in 17 patients, mixed (visceral/somatic and neuropathic) in eight patients, visceral in seven patients, and pure neuropathic in one patient. Twenty-five patients had severe pain ( 7 or more on a numerical scale 0-10). At admission six patients were on non-opioid analgesics, six patients on tramadol, 13 patients on transdermal fentanyl (mean oral morphine equivalents $177 \mathrm{mg}$ ), seven patients on morphine (mean oral morphine equivalents $171 \mathrm{mg}$ ) and three patients on methadone (mean oral morphine equivalents $441 \mathrm{mg}$ ). No significant changes in Karnofsky status were found during the period of study. Tx, defined as time when pain was adequately controlled with stable doses of opioids, occurred $40 \mathrm{~h}$ (range 24-68 h) after admission on average. Although pain levels significantly decreased some hours after starting opioid titration, it was decided to evaluate the time when the pain intensity was optimal for patients and opioid dose was stable. In three patients opioid doses, initially increased were then reduced. OEI was extremely high at $\mathrm{Tx}$, and progressively declined at the following study intervals. Patients were discharged home within a mean of 4.7 days (range 2-7 days).

The pain mechanism did not significantly influenced OEI and pain or symptom intensity. Data regarding pain, symptoms, and other variables examined are listed in Table 1. Pain intensity statistically decreased at Tx, and adequate pain control was maintained for the next 20 days, while maintaining similar opioid doses. Opioid doses at the end of titration were relatively large presenting an OEI of $59 \mathrm{mg}$ per day, which is extremely higher

Table 1

Pain intensity and well being (scale 0-10), symptoms and other variables examined (scale 0-3), expressed as mean (CI 95\%), opioid dose in oral morphine equivalents $(\mathrm{mg})$. Patients who presented a symptom intensity of $2-3$ (scale $0-3$, see text)

\begin{tabular}{lllllr}
\hline & T0 & Tx & T10 & T20 & $\begin{array}{l}\text { Symptom frequency } \\
\text { at intensity 2-3/3 }\end{array}$ \\
\hline Pain intensity & $7.11(6.26-7.97)$ & $2.34(1.82-2.87)^{* * *}$ & $3.06(2.50-3.62)^{* * *, a}$ & $2.82(21.8-3.45)^{* * *}$ \\
Well-being & $4.80(3.90-5.70)$ & $5.06(4.11-6.01)$ & $5.53(4.43-6.63)^{*}$ & $5.36(4.20-6.53)^{*}$ & 9 \\
Nausea-vomiting & $0.66(0.38-0.93)$ & $0.51(0.27-0.76)$ & $0.53(0.28-0.78)$ & $0.45(0.2-0.67)$ & 6 \\
Drowsiness & $0.40(0.21-0.59)$ & $1.0(0.76-1.24)^{* * *}$ & $0.88(0.69-1.07)^{* * *}$ & $0.76(0.56-0.96)^{*, a}$ & 10 \\
Weakness & $1.0(0.76-1.24)$ & $1.03(0.78-1.28)$ & $0.94(0.67-1.20)$ & $0.84(0.53-1.16)$ & 3 \\
Confusion & $0.14(0.0-0.29)$ & $0.26(0.1-0.4)$ & $0.35(0.14-0.56)$ & $0.30(0.10-0.51)$ & 8 \\
Dry mouth & $0.77(0.49-1.05)$ & $1.03(0.80-1.26)$ & $0.76(0.57-0.96)^{\mathrm{a}}$ & $0.73(0.52-0.93)^{\mathrm{a}}$ & 12 \\
Constipation & $0.77(0.41-1.14)$ & $1.11(0.71-1.52)^{*}$ & $1.0(0.58-1.42)$ & $1.03(0.62-1.44)$ & 6 \\
Appetite & $0.71(0.13-1.30)$ & $0.74(0.52-0.97)$ & $0.59(0.36-0.82)$ & $0.61(0.36-0.86)$ & 2 \\
Mood & $0.69(0.50-0.87)$ & $0.60(0.41-0.79)$ & $0.44(0.27-0.62)^{*, a}$ & $0.52(0.31-0.72)$ & $224(205-429)$ \\
Opioid doses & 113 & $231(154-307)$ & $191(120-270)^{*}$ & $2.5(2-7.9)^{\mathrm{b}}$ & \\
OEI mg & & $59(20-102)$ & $7.8(3.1-12)^{\mathrm{b}}$ & $5.9(2.1-8)^{\mathrm{b}}$ & \\
OEI\% & & $52(18-97)$ & $6.9(3-11.8)^{\mathrm{b}}$ &
\end{tabular}

$\mathrm{T} 0=$ data recorded at admission, $\mathrm{Tx}=$ data recorded at the end of opioid titration, $\mathrm{T} 10=$ data recorded 10 days after admission, $\mathrm{T} 20=$ data recorded 20 days after admission (see text).

${ }^{\mathrm{a}} p<0.05$ versus Tx.

$\mathrm{b} p<0.0005$ versus Tx.

${ }^{*} p<0.05$ versus $\mathrm{T} 0$.

*** $p<0.0005$ versus T0. 
in comparison with a previous observation in home care patients (Mercadante et al., 1997). Opioid doses were relatively stable at the following intervals. As a consequence OEI calculated at the following intervals dramatically decreased (Table 1). The symptom more frequently assessed as moderate to severe at admission was weakness, which remained at the same level of intensity after titration and at the following intervals taken into consideration, with a tendency to decrease in time. No significant changes in nausea and vomiting, confusion, and appetite were observed. Nausea and vomiting and confusion of significant intensity were attributed to disease in four and one patients, respectively. Drowsiness intensity significantly increased at Tx, and the other intervals, although tended to decrease at T20. In one patient, a possible concomitant contribution of brain metastases and anticonvulsant drugs, despite other cognitive functions were acceptable, was suggested by the external reviewer. Dry mouth intensity initially tended to increase, and then to maintain similar levels for the period of study. Several concomitant causes of dry-mouth were assessed by the external reviewer, including dehydration, other medication, and mucositis in three, four and two patients, respectively. Constipation intensity significantly increased at Tx, and then was maintained at the same level. Other medications, including anticonvulsants, diuretics, and gastroprotective drugs, were considered as pre-existing causes of constipation in seven patients. Other contributing factors, such as metabolic alterations, dehydration, reduced physical activity, were identified in six patients. Well-being improved at T10 and T20, that is some weeks after achievement of analgesia. However, mood occasionally decreased at T10. The occurrence of opioid-related adverse effects was managed with usual symptomatic treatments. Laxatives, including lactulose and senna, were used routinely. Symptomatic drugs more commonly used are reported in Table 2 . In multivariate analysis, drowsiness and dry mouth were correlated with the opioid doses $(p<0.0005$ and 0.01, respectively). Although 11 patients clearly improved their well-being, and in spite of a trend of correlation between improved well-being and decrease in drowsiness, no significant correlation was found with all other variables examined.

Table 2

Principal symptomatic drugs used during opioid titration

\begin{tabular}{lc}
\hline Drugs & Pts \\
\hline Laxatives & 33 \\
Steroids & 16 \\
Non-opioid analgesics & 15 \\
Antiemetics & 9 \\
Anticonvulsants & 6 \\
Gastroprotectors & 17 \\
\hline
\end{tabular}

\section{Discussion}

\subsection{General considerations}

Patients with severe pain require an aggressive treatment, expecially if they are reporting high levels of pain likely due to poor previous treatment. Timeliness is of paramount importance in these circumstances. Rapid opioid titration, either by oral and/or parenteral route, is advisable, to avoid unnecessary suffering. Morever, pain is known to be associated with poor well-being sensation, decreased appetite, and nausea, and a rapid pain relief may improve some symptoms associated with previous uncontrolled pain. Few studies have dealt with the outcome of pain intervention in patients with severe pain, particularly regarding the consequence of opioidrelated adverse effects. In cancer patients with uncontrolled pain on weak opioids, morphine titration was successful and achieved quickly, with a threefold morphine dose variation and with a little increase in adverse effects. Morphine titration took a mean of 2.3 days, and all patients were stabilized about 6 days after starting morphine. However, these patients had a relatively good pain control ( $32 \mathrm{~mm}$ on VAS), thus the changes of symptoms may be irrelevant (Klepstad et al., 2000b). In an acute setting, pain intensity decreased 14-24 h after the initial pharmacological treatment. Patients had variable levels of pain intensity, and severe pain was assessed in $20 \%$ of patients, with excruciating pain recorded in three patients. In patients with severe pain opioids were prescribed over the phone, before consultation. The effects reported were the result of a therapeutical adjustment, regarding route and drug changes, but opioid doses were not provided, and symptom recording was limited to the first $24 \mathrm{~h}$ (Manfredi et al., 2000). For these reasons data reported in previous studies are not comparable with the design used in this study. Patients with severe pain received an aggressive treatment with escalating doses of opioids, either orally or transdermally assisted by parenteral opioids. This is consistent with the high OEI reported at Tx. While this is more likely to produce a more rapid analgesia, it can also to induce a "jerk" in opioid-related symptoms. In this study, most patients achieved an adequate pain relief within 2 days with a mean dose of oral morphine equivalents of $231 \mathrm{mg}$, and are not comparable with other softer titrations with oral morphine presented in other studies (Klepstad et al., 2000b). Like pain intensity, opioid doses did not significantly increased in the following period.

The occurrence of adverse effects may limit opioid escalation, so they are the most important part of the concept of opioid responsiveness (Mercadante and Portenoy, 2001; Portenoy et al., 1990). Of interest, in the present study, the intensity and the frequency of adverse effects were not the result of the spontaneous 
course of opioid escalation. Rather, they were the consequence of the balanced effect of opioids and illness, and the concomitant symptomatic treatment, which could not be withhold for obvious ethical reasons. This corresponds to the raw clinical scenario where adverse effects occur with a certain intensity to require symptomatic treatment. To reproduce a standard clinical situation, antiemetics and adjuvant analgesics were used in some circumstances to better tolerate opioid effects and improve analgesia, avoiding to use strict protocol limitations. Laxatives were routinely administered. Symptoms were relatively controlled by using these measures, despite the high OEI.

Few studies have dealt with the indirect effects of a significant decrease in pain intensity on other variables, including symptoms. A low increase of adverse effects was associated when starting morphine after weak opioids were unsuccessful. However, this could reflect the characteristics of population chosen, having relatively low level of pain intensity, although a threefold opioid increase was reported (Klepstad et al., 2000a,b). Adverse effects were acceptable even in an acute setting where opioids were prevalently given by intravenous route (Manfredi et al., 2000). The number of opioid attributable adverse effects did not increase over about a 3-week period. However, follow-up study assessment was done at weekly intervals, and data during opioids titration were lacking (Chang et al., 2002).

\subsection{Specific adverse effects}

Central nervous system adverse effects are commonly reported in patients receiving opioids. Drowsiness is observed in $20-60 \%$ of patients receiving oral morphine (Cherny et al., 2001). The symptom more frequently observed during titration was drowsiness, which persisted for 10 days, and then decreased at the end of the study, concluded 20 days after, in comparison to the end titration point $(\mathrm{Tx})$. Considering that opioid doses did not change in the meantime, this means that such dose escalation required relatively more time to induce some tolerance to this adverse effect. The time course of opioid tolerance to some adverse effects has never been established after acute opioid titration with relatively high doses. Unless for one patient, drowsiness observed in this study was exclusively due to opioid effects, as no particular symptomatic treatment was used for this symptom, due to unavailability of a such type of drugs in most European countries. The only method used to limit drowsiness intensity was a more accurate balance between opioid dose and effect. As the symptom was largely distributed in all the patients, the intensity was acceptable in most cases, only six patients presenting moderate or severe drowsiness. In a previous study, the number of patients presenting sedation increased when passing from weak opioids to escalating doses of morphine, the values not changing in the subsequent days when immediate release morphine was substituted with the slow release preparation at the same doses. However, this effect was reported in a short-term period, probably insufficient to reproduce the delayed effects of tolerance (Klepstad et al., 2000b). In a study on the outcome of cancer pain consultation, severe drowsiness was not reported after achieving rapid pain relief in an acute setting, although methylphenidate was added to the therapeutical regime in some patients (Manfredi et al., 2000).

Mild cognitive impairment is common after the initiation of opioid therapy or dose escalation (Bruera et al., 1989), although there is no data on the prevalence of severe delirium (Cherny et al., 2001). Only three patients rated as moderate to severe confusion during opioid titration. This effect was treated by braking opioid escalation or administering symptomatic drugs (the use of haloperidol is included in the list as an antiemetic). Confusion can be also the consequence of poor pain control, rather than being induced by opioid titration. In another acute setting, minimal changes were observed in frequency of delirium after adjusting opioid therapy or changing route of opioid administration (Manfredi et al., 2000).

Data from prospective studies indicate that chronic nausea is observed in $15-30 \%$ of patients receiving oral morphine for chronic cancer pain (Cherny et al., 2001). However, gastrointestinal symptoms can be dependent on progression of disease and numerous factors, including primary diagnosis and concomitant medications. It is difficult to distinguish the prevalent causes during a long-term treatment, as confirmed by possible concomitant factors reported in this study. Nausea and vomiting did not change during titration and remained at acceptable level, probably as the result of antiemetic medications. Only nine patients had an intensity level requiring specific treatment, specifically attributable to opioids. Thus, this symptom can be managed during opioid titration in most cases, although this does not exclude that individual patients be particularly sensible to opioids and resistant to symptomatic treatment. This was confirmed also in an acute setting, where nausea and vomiting were more frequent before consultation than after achieving pain control, 12-24 h after starting the treatment (Manfredi et al., 2000), and when starting oral morphine, either when using immediate or sustained-release preparation (Klepstad et al., 2000b, 2003).

Chronic constipation is observed in $40-70 \%$ of patients receiving oral morphine for chronic cancer pain (Cherny et al., 2001). Constipation significantly increased during opioid titration, despite available standard symptomatic treatment, principally based on senna and lactulose. However, other concomitant causes, particularly the use of other medications, were also screened from 
the documentation. Thus, this symptom should be accurately monitored in the short as well as long-term period and more effective treatments are needed.

Although the level of dry-mouth tended to increase, not significantly, during opioid titration, then it remained at the same level. While it does not exclude that the symptom may be acutely induced and maintained by opioids, the many causes of dry-mouth observed, such as concomitant medication, dehydration, mucositis, frequently reported in advanced cancer patients, make a clear relationship with opioid treatment difficult to prove in the clinical setting.

Opioids, as the spontaneous course of the illness, may induce weakness. It was the symptom more frequently assessed at admission. However, weakness did not change during opioid escalation phase and after achieving pain control. Therefore, it can be supposed that the possible effect of opioids was balanced by the better physical condition fostered by pain control, or that weakness was dependent on the previous status of the illness.

Start of morphine treatment in patients switched from weak opioids had no major influence on aspects of health-related quality of life, other than pain (Klepstad et al., 2000a). A progressive increase of well-being was recorded, particularly after discharge. Significant well-being was perceived 10-20 days after opioid titration, probably as a consequence of a better tolerance to the drug regimen, which did not change significantly. This can also be the consequence of home care setting, as well as an improved tolerance to opioids, not necessarily due to the achievement of pain relief, as no correlation was found with this item. Loss of appetite may be influenced by poor pain control (Klepstad et al., 2000a), or by opioid-induced adverse effects, such as nausea, drowsiness, and weakness. Steroids had been or were administered in about half of patients for weakness and poor appetite, although no single indication was identified. Appetite was not affected by either OEI or achievement of pain relief. Changes in mood levels were difficult to ascertain, as reported in other experiences (Manfredi et al., 2000), and were not related with wellbeing. These data are difficult to interpretate and demonstrate how complex are the factors implicated in mood and appetite, typically influenced by the psychological sphere and the social context. Given these difficulties encountered in the clinical setting, any relationship with pain relief and OEI was impossible to demonstrate. In any case no worsening of these symptoms attributable to opioids was detected.

In this study other more frequently used tools for measuring quality of life (Klepstad et al., 2000a) would have provided more information, but they were not used to avoid a further burden in quite distressed patients. The use of questionnaires with at least 30 items was not considered feasible in this kind of population. This was a preliminary choose, suggested by previous experi- ence in our setting, where more sophisticated instruments were not accepted by patients or data provided were imprecise due to lack of collaboration, also influencing the informed consent for carrying on the study. Therefore, a minimal set was used to gather information on global well-being, appetite, and mood, other than the symptoms regularly assessed for monitoring the patients.

\section{Conclusion}

Data reported in this study are the results of a typical raw clinical scenario presenting in the setting of an acute pain relief and palliative care unit, where patients with severe pain, uncontrolled for prolonged periods of time, are admitted on emergency basis to provide pain relief as soon as possible. Opioid titration in patients with severe pain allows a rapid pain control, without interferring significantly with most symptoms, using available symptomatic therapy. The effects reported are never due exclusively to opioids in this clinical setting, but the consequence of concomitant and symptomatic drugs, actual physical function, and cancer disease per se. This explains the differences in frequencies of opioid-related symptoms reported in literature. Constipation appears the most relevant problem, persisting in time. Weakness is not affected by opioid titration given for achieving optimal pain control. Nausea and vomiting, which are frequently feared symptoms, are effectively controlled by available symptomatic drugs, and do not represent a particular limit in even rapid opioid titration. It is likely that in some cases pain control may reduce these symptoms. The increase in intensity of drowsiness, expected after achieving pain relief, as a consequence of opioid dose increases, but also of a more relaxing situation in patients previously suffering for poor pain control, tended to slowly decrease in time, probably due to the occurrence of tolerance, which can take some weeks to develop after a sharp increase in opioid dose. Well-being may be the late positive effect of pain relief, probably influenced by the setting of home care, or even by the reduction of drowsiness.

It was difficult to establish a clear effect of opioids on mood and appetite, due to methodological limits in the clinical context, where other factors may have a role. Other studies with appropriate design should assess the effects of opioids titration using different opioids, route of administration, or treatment schedules.

\section{References}

Bruera E, MacMillan K, Hanson J, et al. The cognitive effects of the administration of narcotic analgesics in patients with cancer pain. Pain 1989;39:13-6. 
Chang VT, Hwang SS, Kasimis B. Longitudinal documentation of cancer pain management outcomes: a pilot study at a VA Medical Centre. J Pain Symptom Manage 2002;24: 494-505.

Cherny N, Ripamonti C, Pereira J, Davis C, Fallon M, McQuay H, et al. Strategies to manage the adverse effects of oral morphine: an evidence-based report. J Clin Oncol 2001;19:2542-54.

Grond S, Zech D, Diefenbach C, Bischoff A. Prevalence and pattern of symptoms in patients with cancer pain: a prospective evaluation of 1635 cancer patients referred to a pain clinic. J Pain Symptom Manage 1994;9:372-82.

Grond S, Zech D, Diefembach, Radbruck L, lehmann KA. Assessment of cancer pain: a prospective evaluation in 2266 cancer patients referred to a pain service. Pain 1996;64: $107-14$.

Klepstad P, Borchgrevink P, Kaasa S. Effects on cancer patients' health-related quality of life after the start of morphine therapy. J Pain Symptom Manage 2000;20:19-26.

Klepstad P, Kaasa S, Skauge, Borchgrevink P. Pain intensity and side effects during titration of morphine to cancer patients using a fixed schedule dose escalation. Acta Anaesthesiol Scand 2000;44:656-64.
Klepstad P, Kassa S, Jystad A, Hval B, Borchgrevink P. Immediate- or sustained-release morphine for dose finding during start of morphine to cancer patients: a randomized, double-blind trial. Pain $2003 ; 101: 193-8$

Korte W, de Stoutz N, Morant R. Day-to-day titration to initiate transdermal fentanyl in patients with cancer pain: short- and longterm experiences in a prospective study of 39 patients. J Pain Symptom Manage 1996;11:139-46.

Manfredi PL, Chandler S, Pigazzi A, Payne R. Outcome of cancer pain consultations. Cancer 2000;89:920-4.

Mercadante S. Treatment and outcome of cancer pain in advanced cancer patients followed at home. Cancer 1999;85:1849-58.

Mercadante S, Portenoy RK. Opioid poorly responsive cancer pain. Part 1. Clinical considerations. J Pain Symptom Manage 2001;21:144-50.

Mercadante S, Dardanoni G, Salvaggio L, Armata G, Agnello A. Monitoring of opioid therapy in advanced cancer patients. J Pain Symptom Manage 1997;13:204-12.

Portenoy RK, Foley KM, Inturrisi CE. The nature of opioid responsiveness and its implications for neuropathic pain: new hypotheses derived from studies of opioid infusion. Pain 1990;43:273-86. 